\title{
Lignin characterization and catalytic pyrolysis for phenol-rich oil with $\mathrm{TiO}_{2}$-based
}

\section{catalysts}

Zhiguo Dong a, Haiping Yang ${ }^{\text {a* }}$, Peiao Chen ${ }^{\text {a }}$, Zihao Liu ${ }^{\text {a }}$, Yingquan Chen ${ }^{\text {a }}$, Lei Wang

b, Xianhua Wang ${ }^{\mathrm{a}}$ and Hanping Chen ${ }^{\mathrm{a}}$

a State Key Laboratory of Coal Combustion, School of Energy and Power Engineering,

Huazhong University of Science and Technology, Wuhan, 430074, China

b Hubei Provincial Key Laboratory of Green Materials for Light Industry, Hubei University of Technology, Wuhan 430068, China

Table S1. Chemical composition and molecular weight analysis of CSL

\begin{tabular}{ccccccccccc}
\hline \multirow{2}{*}{ Sample } & \multicolumn{8}{c}{ Proximate analysis (wt.\%)/d } & \multicolumn{7}{c}{ Ultimate analysis (wt.\%)/daf } & \multirow{2}{*}{$\mathrm{H} / \mathrm{C}^{\mathrm{b}}$} & $\mathrm{O} / \mathrm{C}^{\mathrm{b}}$ \\
\cline { 2 - 9 } & $\mathrm{A}$ & $\mathrm{V}$ & $\mathrm{FC}$ & $\mathrm{C}$ & $\mathrm{H}$ & $\mathrm{N}$ & $\mathrm{S}$ & $\mathrm{O}^{*}$ & \\
\hline \multirow{3}{*}{$\mathrm{CSL}$} & 0.85 & 55.34 & 43.81 & 65.56 & 4.91 & 1.11 & 0.11 & 28.30 & 0.90 & 0.32 \\
\cline { 2 - 9 } & $\mathrm{M}_{\mathrm{n}} / \mathrm{g} \cdot \mathrm{mol}^{-1}$ & $\mathrm{M}_{\mathrm{w}} / \mathrm{g} \cdot \mathrm{mol}^{-1}$ & $\mathrm{M}_{\mathrm{w}} / \mathrm{M}_{\mathrm{n}}$ & $\mathrm{M}_{\mathrm{r}} / \mathrm{g} \cdot \mathrm{mol}^{-1}$ & $\mathrm{C}_{9}$ molecular formula & $\mathrm{M}_{\mathrm{w}} / \mathrm{M}_{\mathrm{r}}$ \\
\cline { 2 - 9 } & 12214 & 16121 & 1.32 & 162.71 & $\mathrm{C}_{9} \mathrm{H}_{8.09} \mathrm{O}_{2.91}$ & 99 \\
\hline
\end{tabular}

* Corresponding author

E-mail address: yhping2002@163.com (Haiping Yang) 
Table S2. Assignment of main signals in the HSQC spectra of the CSL

\begin{tabular}{|c|c|c|}
\hline Lable & $\delta \mathrm{C} / \delta \mathbf{H}(\mathbf{p p m})$ & Assignments \\
\hline $\mathrm{OMe}$ & $55.5 / 3.74$ & $\mathrm{C}-\mathrm{H}$ in methoxyls \\
\hline $\mathrm{A}_{\alpha}$ & $71.75 / 4.84$ & $\mathrm{C}_{\alpha}-\mathrm{H}_{\alpha}$ in $\beta-\mathrm{O}-4$ unit (A) (Erythro) \\
\hline $\mathrm{A}_{\gamma}$ & $58.47 / 3.23$ & $\mathrm{C}_{\gamma}-\mathrm{H}_{\gamma}$ in $\beta-\mathrm{O}-4$ substructures (A) \\
\hline $\mathrm{A}_{\beta}(\mathrm{G})$ & $83.76 / 4.4$ & $\mathrm{C}_{\beta}-\mathrm{H}_{\beta}$ in $\beta-\mathrm{O}-4$ linked to $\mathrm{G}(\mathrm{A})$ \\
\hline $\mathrm{A}_{\beta}(\mathrm{S})$ & $86.33 / 4.08$ & $\mathrm{C}_{\beta}-\mathrm{H}_{\beta}$ in $\beta-\mathrm{O}-4$ linked to $\mathrm{S}$ (A, Erythro) \\
\hline $\mathrm{B}_{\alpha}$ & $87.63 / 5.58$ & $\mathrm{C}_{\alpha}-\mathrm{H}_{\alpha}$ in phenylcoumaran (B) \\
\hline $\mathrm{C}_{\alpha}$ & $81.82 / 4.9$ & $\mathrm{C}_{\alpha}-\mathrm{H}_{\alpha}$ in spirodienones $(\mathrm{C})$ \\
\hline $\mathrm{D}_{\alpha}$ & $78.44 / 5.58$ & $\mathrm{C}_{\alpha}-\mathrm{H}_{\alpha}$ in $\alpha, \beta$-diaryl ethers (D) \\
\hline $\mathrm{T}_{3}$ & $107.04 / 6.76$ & $\mathrm{C}_{3}-\mathrm{H}_{3}$ in tricin $(\mathrm{T})$ \\
\hline $\mathrm{T}_{6}$ & $98.53 / 6.38$ & $\mathrm{C}_{2,6}-\mathrm{H}_{2,6}$ in tricin $(\mathrm{T})$ \\
\hline $\mathrm{T}_{2,6}^{\prime}$ & $104.14 / 7.34$ & $\mathrm{C}_{2,6}^{\prime}-\mathrm{H}_{2,6}^{\prime}$ in tricin $(\mathrm{T})$ \\
\hline $\mathrm{G}_{2}$ & $111.27 / 6.82$ & $\mathrm{C}_{2}-\mathrm{H}_{2}$ in guaiacyl units $(\mathrm{G})$ \\
\hline $\mathrm{G}_{5}$ & $115.06 / 6.8$ & $\mathrm{C}_{5}-\mathrm{H}_{5}$ in guaiacyl units $(\mathrm{G})$ \\
\hline $\mathrm{G}_{6}$ & $119.01 / 6.68$ & $\mathrm{C}_{6}-\mathrm{H}_{6}$ in guaiacyl units $(\mathrm{G})$ \\
\hline $\mathrm{S}_{2,6}$ & $104 / 6.65$ & $\mathrm{C}_{2,6}-\mathrm{H}_{2,6}$ in syringyl units $(\mathrm{S})$ \\
\hline $\mathrm{S}_{2,6}^{\prime}$ & $106.49 / 7.3$ & $\mathrm{C}_{2,6}-\mathrm{H}_{2,6}$ in oxidized $\mathrm{S}$ units $\left(\mathrm{S}^{\prime}\right)$ \\
\hline $\mathrm{H}_{2,6}$ & $128.37 / 7.02$ & $\mathrm{C}_{2,6}-\mathrm{H}_{2,6}$ in $\mathrm{H}$ units $(\mathrm{H})$ \\
\hline $\mathrm{PCE}_{2,6}$ & $130.03 / 7.48$ & $\mathrm{C}_{2,6}-\mathrm{H}_{2,6}$ in $p$-coumarate $(p-\mathrm{CE})$ \\
\hline $\mathrm{PCE}_{7}$ & $144.07 / 7.47$ & $\mathrm{C}_{7}-\mathrm{H}_{7}$ in $p$-coumarate $(p-\mathrm{CE})$ \\
\hline $\mathrm{PCE}_{8}$ & $115.34 / 6.24$ & $\mathrm{C}_{8}-\mathrm{H}_{8}$ in $p$-coumarate $(p-\mathrm{CE})$ \\
\hline $\mathrm{FA}_{2}$ & $111.74 / 7.35$ & $\mathrm{C}_{2}-\mathrm{H}_{2}$ in ferulate $(p-\mathrm{FA})$ \\
\hline $\mathrm{FA}_{6}$ & $123.18 / 7.12$ & $\mathrm{C}_{6}-\mathrm{H}_{6}$ in ferulate $(p$-FA) \\
\hline
\end{tabular}


Table S3. Calibration results of main phenols

\begin{tabular}{lll}
\hline \multirow{2}{*}{ Compound } & \multicolumn{1}{l}{$(\mathbf{m g} / \mathbf{m l})$} & $=\mathbf{a}+\mathbf{b X} \quad$ (peak \\
& $\mathbf{a r e a )}$ & \\
\cline { 2 - 3 } & $\mathbf{a}$ & $\mathbf{b}$ \\
\hline Phenol & 0.08959 & $2.68 \mathrm{E}-09$ \\
p-Cresol & 0.11897 & $2.53 \mathrm{E}-09$ \\
Phenol, 4-ethyl- & 0.13434 & $1.8 \mathrm{E}-09$ \\
Phenol, 4-vinyl- & 0.09 & $2.67 \mathrm{E}-09$ \\
Phenol, 2-methoxy- & 0 & $2.61 \mathrm{E}-09$ \\
Phenol, 2,6-dimethoxy- & 0.08415 & $2.36 \mathrm{E}-09$ \\
\hline
\end{tabular}


Table S4. Yields of phenolic compounds (in $\mathrm{mg} / \mathrm{g}$ lignin) obtained with different metal loading of $\mathrm{Mo} / \mathrm{TiO}_{2}$ catalyst with lignin at $450{ }^{\circ} \mathrm{C}$.

\begin{tabular}{clllll}
\hline $\begin{array}{c}\text { RT } \\
\text { (min) }\end{array}$ & Compound & $\mathbf{5 \% M o}$ & $\mathbf{1 0 \% M o}$ & $\mathbf{2 0 \% M o}$ & $\mathbf{M o O}_{3}$ \\
\hline 10.82 & Phenol & 1.55 & 1.90 & 1.79 & 0.90 \\
13.09 & Phenol, 2-methyl- & 1.19 & 1.19 & 1.19 & 1.19 \\
13.88 & p-Cresol & 1.19 & 1.31 & 1.30 & 1.19 \\
16.30 & Phenol, 2,4-dimethyl- & 1.34 & 1.34 & 1.44 & 1.34 \\
17.21 & Phenol, 4-ethyl- & 1.76 & 2.29 & 1.97 & 1.57 \\
19.05 & Phenol, 4-vinyl- & 1.93 & 3.93 & 2.29 & 2.14 \\
& Total monophenols & $\mathbf{8 . 9 6}$ & $\mathbf{1 1 . 9 5}$ & $\mathbf{9 . 9 9}$ & $\mathbf{8 . 3 3}$ \\
14.05 & Phenol, 2-methoxy- & 1.54 & 2.36 & 1.73 & 0.74 \\
20.32 & Phenol, 4-ethyl-2-methoxy- & 0.99 & 1.48 & 1.26 & 0.74 \\
17.92 & Creosol & 0.63 & 0.86 & 0.75 & 0.71 \\
21.19 & 2-Methoxy-4-vinylphenol & 1.34 & 2.62 & 1.73 & 1.38 \\
22.22 & Phenol, 2-methoxy-4-propyl- & 0.00 & 0.50 & 0.00 & 0.00 \\
& Total guaiacols & $\mathbf{4 . 5 0}$ & $\mathbf{7 . 8 2}$ & $\mathbf{5 . 4 8}$ & $\mathbf{3 . 5 7}$ \\
21.96 & Phenol, 2,6-dimethoxy- & 3.02 & 3.55 & 3.06 & 1.97 \\
23.65 & 3,5-Dimethoxy-4-hydroxytoluene & 1.53 & 1.73 & 1.77 & 1.82 \\
27.10 & (E)-2,6-Dimethoxy-4-(prop-1-en-1-yl)phenol & 1.47 & 1.67 & 1.46 & 1.48 \\
& Total syringols & $\mathbf{6 . 0 2}$ & $\mathbf{6 . 9 5}$ & $\mathbf{6 . 2 9}$ & $\mathbf{5 . 2 6}$ \\
& Total phenols & $\mathbf{1 9 . 4 8}$ & $\mathbf{2 6 . 7 2}$ & $\mathbf{2 1 . 7 6}$ & $\mathbf{1 7 . 1 6}$ \\
\hline
\end{tabular}




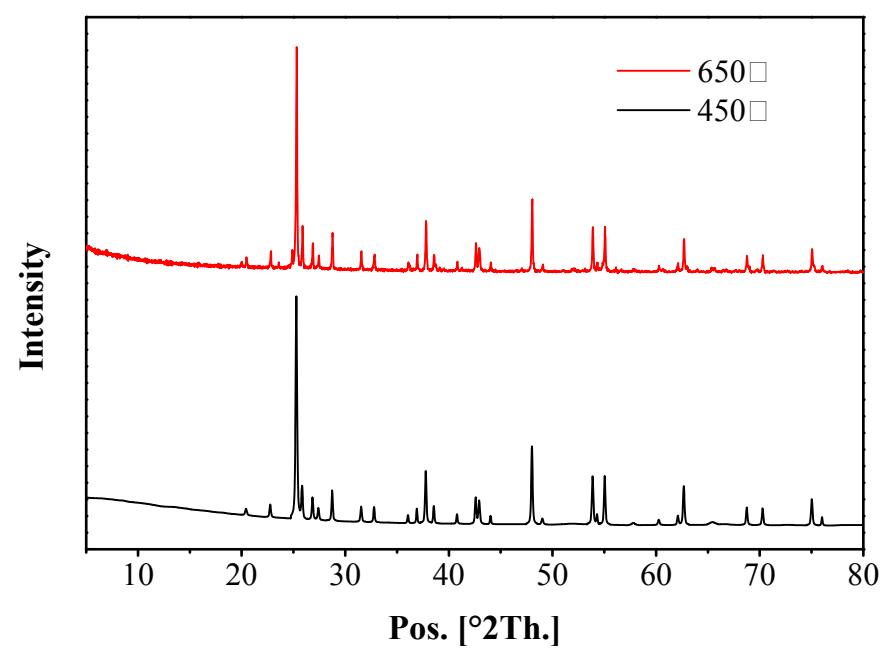

Figure S1. XRD spectra of $\mathrm{Mo} / \mathrm{TiO}_{2}$ calcined at different temperatures 


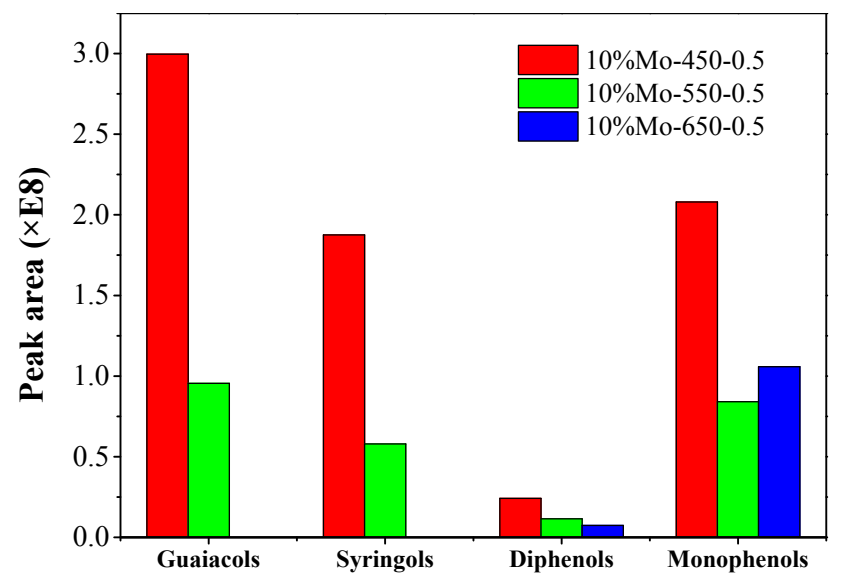

Figure S2. Effect of temperature on phenolic yield of $\mathrm{Mo} / \mathrm{TiO}_{2}$ catalyzed lignin pyrolysis 